Bull. Austral. Math. Soc.

VoL. 59 (1999) [203-215]

\title{
SOME RESULTS ON DEFINING SETS OF $t$-DESIGNS
}

\author{
Brenton D. Gray and Colin Ramsay
}

\begin{abstract}
We investigate how varying the parameters of $t-(v, k, \lambda)$ designs affects the sizes of smallest defining sets. In particular, we consider the effect of varying each of the parameters $t, v$ and $\lambda$. We establish a number of new bounds for the sizes of smallest defining sets and find the size of smallest defining sets for an infinite family of designs. We also show how one of our results can be applied to the problem of finding critical sets of Latin squares.
\end{abstract}

\section{INTRODUCTION}

Let $V$ be a $v$-set, and suppose that $\mathcal{B}$ is a collection of $k$-subsets of $V$ with the property that each $t$-subset of $V$ is in exactly $\lambda$ of the elements of $\mathcal{B}$. Then the ordered pair $(V, \mathcal{B})$ is called a $t-(v, k, \lambda)$ design. The elements of $V$ are called points, and the elements of $\mathcal{B}$ blocks. We often abbreviate $t$ - $(v, k, \lambda)$ design to $t$-design, or simply design. A design with $\lambda=1$ is called a Steiner design.

To avoid trivialities, we assume throughout that $0<t<k<v-t$. Although a block is formally defined as a set of elements, it is often simpler to avoid explicitly using set notation. For instance, the block $\{1,3, a, b\}$ may instead be written as $\{13 a b\}$ or $13 a b$. Further, although designs may be multisets, we use set notation and terminology throughout.

The concept of a defining set of a $t$-design was introduced by $\mathrm{K}$. Gray in the series of papers $[6,7,8]$; see also the survey papers by Street $[\mathbf{2 3}, \mathbf{2 4}]$.

DEFinition 1.1: A set of blocks $S$ which is a subset of a unique $t-(v, k, \lambda)$ design $D$ is a defining set of $D$. The size of $S$ equals $|S|$ and $S$ is said to be smallest if no other defining set of $D$ has smaller size. A defining set is minimal if it does not properly contain a defining set.

EXAmple 1.2. Let $S=\{124,235,346\}$. Then it is easily verified that $S$ is a smallest defining set of the $2-(7,3,1)$ design $D=(V, \mathcal{B})$ with $V=\{1,2,3,4,5,6,7\}$ and $\mathcal{B}=$ $\{124,235,346,457,561,672,713\}$. The design $D$ is known as a Fano plane.

Received 26th June, 1998

The first author was supported by an Australian Postgraduate Award and ARC Grant A49532477. The second author was supported by an Australian Postgraduate Award.

Copyright Clearance Centre, Inc. Serial-fee code: 0004-9729/99 \$A2.00+0.00. 
A number of authors have discussed the sizes of smallest defining sets of families of designs. Let $\left\{D_{d}\right\}_{d \in \Lambda}$ denote a family of designs with some indexing set $\Lambda$. Let $\mu_{d}$ denote the fraction of blocks in a smallest defining set of $D_{d}$. B. Gray, Hamilton and O'Keefe [5] have shown that if $D_{d}=P G(2, d)$, then $\mu_{d} \leqslant 1 / 2+\varepsilon_{d}$, where $\varepsilon_{d} \rightarrow 0$ as $d \rightarrow \infty$. In contrast, B. Gray [4] has shown that if $D_{d}$ is the symmetric design obtained from the points and hyperplanes of $P G(d, 2)$, then $\mu_{d} \rightarrow 1$ as $d \rightarrow \infty$. If $d$ is an odd prime power and $D_{d}$ is the Hadamard design cyclically generated from the quadratic residues of $G F(d)$, then Sarvate and Seberry [22] conjecture that $\mu_{d} \leqslant 1 / 2$.

In all of these families of designs the block size $k$ varies. In this paper we investigate how varying each of the parameters $t, \lambda$ and $v$, whilst $k$ remains fixed, affects the sizes of smallest defining sets.

The background material we require will be reviewed in the next section. In the remainder of this section we summarise our results and the layout of the remaining sections.

The number of blocks, $|\mathcal{B}|$, in a design is denoted by $b$, and each point appears in exactly $r$ blocks. Standard counting arguments yield the following result.

LEMma 1.3. For a $t$ - $(v, k, \lambda)$ design $D$, the following relations hold:

$$
r v=b k \text { and } \lambda\left(\begin{array}{l}
v \\
t
\end{array}\right)=b\left(\begin{array}{l}
k \\
t
\end{array}\right) \text {. }
$$

Further, $D$ is an $s-\left(v, k, \lambda_{s}\right)$ design for $0 \leqslant s \leqslant t$, and the values of $\lambda_{s}$ are given by

$$
\lambda_{s}=\lambda\left(\begin{array}{l}
v-s \\
t-s
\end{array}\right) /\left(\begin{array}{c}
k-s \\
t-s
\end{array}\right) .
$$

Note that $\lambda_{0}=b, \lambda_{1}=r$ and $\lambda_{t}=\lambda$.

We show in Section 3 that if $s_{t}$ and $s_{s}$ are the sizes of a smallest defining set of a design $D$ considered as a $t$-design and as an $s$-design respectively, then $s_{s} \geqslant s_{t}+2^{t-s}-1$.

In Section 4, we present some new bounds for the size of a smallest defining set of a $t-(v, k, \lambda)$ design, when $k=t+1$ or $v=k+t+1$. Using these results, we completely solve for the sizes of smallest defining sets of the infinite family of $2-(6,3, \lambda)$ designs. We also investigate the infinite families of $2-(7,3, \lambda)$ and $3-(8,4, \lambda)$ designs.

Definition 1.4: Let $D=(V, \mathcal{B})$ and suppose $S \subseteq \mathcal{B}$. If $S \subseteq \mathcal{C}$, where $E=(V, \mathcal{C})$ is a design with the same parameters as $D$, implies that:

1. $E$ is isomorphic to $D$, then $S$ is said to be a class defining set of $D$;

2. $E=D$ or $E$ is not isomorphic to $D$, then $S$ is said to be a member defining set of $D$.

Note that $S$ is a defining set of $D$ if and only if $S$ is both a class and a member defining set of $D$.

Let $\left\{D_{v}\right\}$ be a family of Steiner $t-(v, k, 1)$ designs, where $k$ and $t$ are fixed. In Section 5 we show that, as $v \rightarrow \infty$, the fraction of blocks contained in a smallest member 
defining set of $D_{v}$ approaches zero. As a corollary to this, the sizes of smallest class defining sets and smallest defining sets are asymptotically equal. We discuss the implications of an analogue of this result for critical sets in Latin squares.

\section{BACKGROUND}

Let $S_{v}$ denote the symmetric group of permutations on a $v$-set. Two designs, $D_{1}=$ $\left(V, \mathcal{B}_{1}\right)$ and $D_{2}=\left(V, \mathcal{B}_{2}\right)$, are said to be isomorphic if there exists $\rho \in S_{v}$ such that $\rho \mathcal{B}_{1}=\mathcal{B}_{2}$. If no such $\rho$ exists, then $D_{1}$ and $D_{2}$ are non-isomorphic. If $\mathcal{B}_{1} \neq \mathcal{B}_{2}$, then $D_{1}$ and $D_{2}$ are said to be distinct. If all distinct $t-(v, k, \lambda)$ designs are isomorphic, we say that the design is unique.

Given a design $D$, if $\rho \in S_{v}$ is such that $\rho \mathcal{B}=\mathcal{B}$, then $\rho$ is called an automorphism of $D$. The set of all automorphisms of $D$ is a subgroup of $S_{v}$, and is denoted by aut $(D)$. If $D$ is a $t-(v, k, \lambda)$ design, then the class of designs isomorphic to $D$ has size $\left|S_{v}\right| /|\operatorname{aut}(D)|=$ $v ! /|\operatorname{aut}(D)|$.

Definition 2.1: A $t$ - $(v, k, \lambda)$ design whose blocks can be partitioned into sets in such a way that each set is a $t-\left(v, k, \mu_{i}\right)$ design, where $\sum_{i} \mu_{i}=\lambda$ and $0<\mu_{i}<\lambda$, is called decomposable. If no such partition exists, the design is indecomposable.

For given $t, v$ and $k$ the number of indecomposable designs is finite (Engel [3]). This number is not known, in general. However, when all the indecomposable designs are known, all $t-(v, k, \lambda)$ designs can be constructed (Grüttmüller [11]).

EXAMPLE 2.2. Let $V=\{1,2,3,4,5,6,7\}, D=(V, \mathcal{B})$ and $I=(V, \mathcal{I})$, where

$$
\begin{aligned}
\mathcal{B}= & \{124,235,346,457,561,672,713\} \cup\{124,135,346,457,562,671,723\} \\
& \cup\{125,134,356,457,462,671,723\}, \\
\mathcal{I}= & \{123,125,127,135,136,145,146,147,167,234,236,246,247,256,257, \\
& 345,347,357,367,456,567\} .
\end{aligned}
$$

Then $D$ is a $2-(7,3,3)$ design whose blocks can be partitioned into Fano planes; thus $D$ is decomposable. However, the $2-(7,3,3)$ design $I$ is indecomposable.

Lemma 2.3. (K. Gray [6]) Let $D=D_{1} \cup D_{2}$ be a $t$ - $\left(v, k, \mu_{1}+\mu_{2}\right)$ design, where $D_{1}$ and $D_{2}$ are $t-\left(v, k, \mu_{1}\right)$ and $t-\left(v, k, \mu_{2}\right)$ designs respectively. Let $s, s_{1}$ and $s_{2}$ denote the sizes of smallest defining sets of $D, D_{1}$ and $D_{2}$ respectively. Then $s \geqslant s_{1}+s_{2}$.

Notation 2.4. If $A$ is a collection of blocks, then

$$
A^{x}=\{B \backslash\{x\}: x \in B \text { and } B \in A\} \text { and } \overline{A^{x}}=\{B: x \notin B \text { and } B \in A\} .
$$

If $A$ is a set of blocks and $x$ is a new element not in any block of $A$, then we use the notation $x A$ to stand for the set of blocks $\{\{x\} \cup B: B \in A\}$.

We say that $x$ has multiplicity $r_{x}$ in $A$ if $x$ is contained in $r_{x}$ blocks of $A$; that is, $r_{x}=\left|A^{x}\right|$. Suppose that $D=(V, \mathcal{B})$ is a $t-(v, k, \lambda)$ design, and let $x$ be any point in $V$. 
The restriction of $D$ on $x$ is the $(t-1)-(v-1, k-1, \lambda)$ design $\left(V \backslash\{x\}, \mathcal{B}^{x}\right)$. Suppose that $x$ is a new point not in $V$. Then it may be possible to extend $D$ to a $(t+1)$ $(v+1, k+1, \lambda)$ design $(V \cup\{x\}, x \mathcal{B} \cup A)$, called an extension of $D$, for some set of blocks $A$. If $A$ is the complement of $x \mathcal{B}$ (with respect to $V \cup\{x\}$ ), then the process of extension is called extension by complementation. The set of blocks of an extended design formed by complementation is necessarily self-complementary.

Let $V$ be a $v$-set and $T_{1}, T_{2}$ be collections of $m k$-subsets of $V$. We say that $T_{1}$ and $T_{2}$ are $t$-balanced if each $t$-subset of $V$ is contained in the same number of blocks of $T_{1}$ and of $T_{2}$. If $T_{1}$ and $T_{2}$ are disjoint and $t$-balanced, then $T=T_{1}-T_{2}$ is said to be a $(v, k, t)$ trade of volume $m(T)=m$. The set of elements of $V$ contained in $T_{1}$ is called the foundation, denoted by $F\left(T_{1}\right)$. Note that $F\left(T_{1}\right)=F\left(T_{2}\right)$, and so we define $F(T)=F\left(T_{1}\right)$. We write $f(T)$ for $|F(T)|$.

In $T=T_{1}-T_{2}$, '-' does not represent the set-difference binary operation, which is always represented by ' $\backslash$ '. We think of the blocks of $T_{1}$ as being labelled ' + ' and those of $T_{2}$ as being labelled '-'. If $T=T_{1}-T_{2}$ is a $(v, k, t)$ trade, we often refer to the single collection $T_{1}$ as a trade. If $D=(V, \mathcal{B})$ is a $t-(v, k, \lambda)$ design with $T_{1} \subseteq \mathcal{B}$, then the design is said to contain the trade. If the value of $v$ is not of interest, we often speak of a $(k, t)$ trade instead of a $(v, k, t)$ trade. If $T_{1}=T_{2}=\emptyset$, then the trade is said to be void; we ignore void trades in what follows.

EXAMPLE 2.5. $T=T_{1}-T_{2}=+135+146+236+245-136-145-235-246$ is a $(6,3,2)$ trade, with $F(T)=\{1,2,3,4,5,6\}, f(T)=6$ and $m(T)=4$.

Trades, which are also known as null $t$-designs, have many uses in the theory of designs. For example, they can be used to construct $t$-designs with different support sizes (Hedayat [12]) and are related to the design intersection problem (Billington [1]). Of relevance to us is the relationship between trades and defining sets of designs.

Theorem 2.6. ([6]) Suppose $D=(V, \mathcal{B})$ and $S \subseteq \mathcal{B}$. Then $S$ is a defining set of $D$ if and only if $S$ intersects each trade in $D$.

Proof: Suppose $S$ is a defining set of $D$. If $T_{1}$ is a trade in $D$, then $S \cap T_{1} \neq \emptyset$, else $S$ is also a subset of the design with blocks $\left(\mathcal{B} \backslash T_{1}\right) \cup T_{2}$, where $T_{1}-T_{2}$ is a trade.

Conversely, suppose $S \subseteq \mathcal{B}$ intersects each trade in $D$. If $S$ is not a defining set of $D$, then $S \subseteq D_{2}$ for some design $D_{2}$ with the same parameters as, but distinct from, $D$. Let $T_{1}$ comprise the blocks of $D$ not in $D_{2}$ and $T_{2}$ comprise the blocks of $D_{2}$ not in $D$. Then $T_{1}-T_{2}$ is a trade, with $T_{1}$ in $D$. Furthermore, $S$ is disjoint from $T_{1}$, which is a contradiction.

The final results we shall need give some simple properties of trades. Note that Lemma 2.8 follows from Lemma 2.7(1).

LEMma 2.7. ([12], Hwang [13]) Let $T=T_{1}-T_{2}$ be a non-void $(k, t)$ trade. Then: 
(1) $T$ is a $(k, s)$ trade for all $0<s<t$;

(2) $m(T) \geqslant 2^{t}$;

(3) $f(T) \geqslant k+t+1$.

Lemma 2.8. ([13]) Suppose that $T=T_{1}-T_{2}$ is a $(k, t)$ trade. Then:

(1) $T^{x}=T_{1}^{x}-T_{2}^{x}$ is a $(k-1, t-1)$ trade of volume $r_{x}$;

(2) $\overline{T^{x}}=\overline{T_{1}^{x}}-\overline{T_{2}^{x}}$ is a $(k, t-1)$ trade of volume $m(T)-r_{x}$;

(3) $x T^{x}=x T_{1}^{x}-x T_{2}^{x}$ is a $(k, t-1)$ trade of volume $r_{x}$.

\section{VARYING $t$}

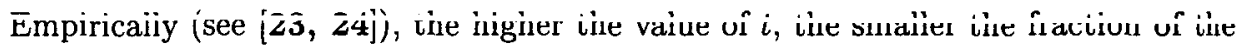
blocks needed to define a design. A classical example of this fact is the result by Curtis [2] that only eight out of the 759 blocks are needed to define the unique $5-(24,8,1)$ Matthieu design.

Little is known in general about the effect on the sizes of defining sets in a $t$-design of varying $t$. K. Gray [6] showed that the size of a smallest defining set of the unique $3-(8,4,1)$ design is three, whereas when considered as one of the four $2-(8,4,3)$ designs the size of a smallest defining set is six. Greenhill and Street [9] showed that the size of a smallest defining set of the unique 3- $(10,4,1)$ design is four, whereas the size of a smallest defining set is sixteen when this design is considered as a $2-(10,4,4)$ design (of which there are more than $1.7 \times 10^{6}$, see Mathon and Rosa [19]).

Lemma 3.1. Let $D$ be a $t-\left(v, k, \lambda_{t}\right)$ design. For $0<s<t, D$ is also an $s-\left(v, k, \lambda_{s}\right)$ design where $\lambda_{s}$ and $\lambda_{t}$ are related as in Lemma 1.3. If $S$ is a defining set of $D$ considered as an $s$-design, then $S$ is also a defining set of $D$ considered as a $t$-design.

Proof: By Lemma 2.7(1) any $(k, t)$ trade in $D$ is also a $(k, s)$ trade in $D$ and the result follows from Theorem 2.6.

Thus, if $s_{t}$ and $s_{s}$ are the sizes of smallest defining sets of $D$ considered as a $t$-design and as an $s$-design respectively, then $s_{t} \leqslant s_{s}$. By considering the structure of trades and of defining sets, we now show that equality is not possible.

LEMma 3.2. If $T$ is a non-void $(k, t)$ trade, and $0<s<t$, then the blocks of $T$ can be partitioned into at least $2^{t-s}$ non-void $(k, s)$ trades.

Proof: Clearly there exists $x \in F(T)$ such that $r_{x} \neq m(T)$. By Lemma 2.8, $x T^{x}$ and $\overline{T^{x}}$ are two non-void $(k, t-1)$ trades which partition the blocks of $T$. So $T$ can be partitioned into two $(k, t-1)$ trades. The result now follows by induction.

Lemma 3.3. Let $M$ be a minimal defining set of a $t$-design $D$. Then there exists a trade $T^{M}$ in $D$ such that $\left|M \cap T^{M}\right|=1$. 
Proof: As $M$ is a defining set of $D$, certainly $|M \cap T| \geqslant 1$ for every trade $T$ in $D$. Suppose that $|M \cap T| \geqslant 2$ for every trade $T$ in $D$. Then, if $B$ is any block in $M$, $|(M \backslash\{B\}) \cap T| \geqslant 1$ for every trade $T$ in $D$ and $M \backslash\{B\}$ is also a defining set of $D$. This is a contradiction, since $M$ is minimal.

THEOREM 3.4. Let $D$ be as in Lemma 3.1. Let $s_{t}$ and $s_{s}$ be the sizes of smallest defining sets of $D$ considered as a $t$-design and as an $s$-design respectively. Then

$$
s_{s} \geqslant s_{t}+2^{t-s}-1 \text {. }
$$

Proof: Let $S$ be a smallest defining set of $D$ considered as an $s$-design. Then, by Lemma 3.1, $S$ is also a defining set of $D$ considered as a $t$-design. Hence there exists $M \subseteq S$ such that $M$ is a minimal defining set of $D$ considered as a $t$-design. We show that

$$
|S| \geqslant|M|+2^{t-s}-1
$$

The result then follows immediately, as $|S|=s_{s}$ and $|M| \geqslant s_{t}$.

To prove (*), we first note that if $T$ is a $(k, t)$ trade in $D$, then $|S \cap T| \geqslant 2^{t-s}$ by Lemma 3.2. But by Lemma 3.3, if $M \subseteq S$ is a minimal defining set of $D$ considered as a $t$-design, then $\left|M \cap T^{M}\right|=1$ for some $(k, t)$ trade $T^{M}$ in $D$. Thus $M$ has at least $2^{t-s}-1$ less blocks than $S$.

As the results quoted earlier for the $3-(8,4,1)$ and $3-(10,4,1)$ designs illustrate, the bound of Theorem 3.4 is certainly not tight. To conclude this section we show that, considered as 1-designs, Steiner designs need all but one of their blocks to define them. Thus the $3-(8,4,1)$ and $3-(10,4,1)$ designs, considered as 1-designs, have smallest defining sets of 13 and 29 blocks respectively.

Lemma 3.5. Let $D=(V, \mathcal{B})$ be a $t-(v, k, 1)$ design. Then, considered as a 1 design, $D$ has a smallest defining set size of $b-1$.

Proof: Since $D$ is Steiner and $k \geqslant t+1$, then any two blocks in $\mathcal{B}$ intersect in at most $k-2$ points. It is easy to see that any two blocks of size $k$ that share at most $k-2$ points must form one half of some $(k, 1)$ trade. The result now follows from Theorem 2.6 .

\section{VARYING $\lambda$}

In this section we investigate the sizes of smallest defining sets of some families of designs in which $\lambda$ varies. By combining some new upper bounds on the size of smallest defining sets with information on the designs' decomposability, we solve for the sizes of smallest defining sets of the $2-(6,3, \lambda)$ designs and obtain good bounds on the sizes for the $2-(7,3, \lambda)$ and $3-(8,4, \lambda)$ designs.

LEMMA 4.1. The size $s$ of a smallest defining set of a $t-\left(v, t+1, \lambda_{t}\right)$ design, $D=(V, \mathcal{B})$, with $b$ blocks satisfies $s \leqslant b-r$. 
Proof: Let $e \in V$ and let $S$ be the collection of $b-r$ blocks of $D$ which do not contain $e$. If $W$ is a $t$-subset not containing $e$ which does not occur $\lambda_{t}$ times in the blocks of $S$, then $S$ forces the block(s) $\mathrm{eW}$. In this way, all the $r$ blocks of $D$ containing $e$ are forced and $S$ is a defining set of $D$.

Lemma 4.2. Let $D=(V, \mathcal{B})$ be a $t-\left(k+t+1, k, \lambda_{t}\right)$ design. For $x \in V$, let $D^{x}$ be the $(t-1)-\left(k+t, k-1, \lambda_{t}\right)$ design $\left(V \backslash\{x\}, \mathcal{B}^{x}\right)$ (so $D^{x}$ is the restriction of $D$ on $x$ ). Suppose that $s$ and $s^{x}$ are the sizes of smallest defining sets of $D$ and $D^{x}$ respectively. Then $s \leqslant s^{x} \leqslant r$.

Proof: That $s^{x} \leqslant r$ is trivial. Let $S^{x}$ be a smallest defining set of $D^{x}$, and set $S=x S^{x}$. We show that $S$ is a defining set of $D$.

Certainiy $\tilde{S}$ iorces the $r$ biocks oî $\bar{D}$ which conidin ine eienteni $x$. Tíne remaining $b-r$ blocks of $D$ cannot contain a $(k, t)$ trade as the foundation of such a trade would be strictly less than $k+t+1$, contradicting Lemma 2.7(3).

EXAMPLE 4.3. The size of a smallest defining set of a $4-(11,5,1)$ design is five. It follows from the previous lemma that the size of a smallest defining set of a $5-(12,6,1)$ design is at most five. It is, in fact, straightforward to see that this size must equal five; see [9].

Suppose $k=t+1$ and $v=k+t+1$, so that $r=b / 2$. Lemmas 4.1 and 4.2 each imply that $s \leqslant r$. However, we can improve this bound by combining the approaches of the two lemmas.

LEMMA 4.4. The size $s$ of a smallest defining set of a $t-\left(2 t+2, t+1, \lambda_{t}\right)$ design, $D=(V, \mathcal{B})$, satisfies $s \leqslant r-\lambda_{2}$.

Proof: Let $a, b \in V$. Partition $\mathcal{B}$ into sets $X, Y$ and $Z$ where $X$ consists of the blocks of $D$ that contain $a$ but not $b, Y$ consists of the blocks that contain $a$ and $b$, and $Z$ consists of the blocks that do not contain $a$.

Now given $X, Y$ is forced since any $t$-subset that contains $a$ but not $b$ and which has not occurred $\lambda_{t}$ times in $X$ must form a block with $b$ in $Y$. Thus $X$ forces $X \cup Y$, which is a defining set of $D$ as in the proof of Lemma 4.2.

THEOREM 4.5. Let $\lambda \geqslant 2$ be even, $J$ be a $2-(6,3, \lambda)$ design, $s_{\lambda}$ be the size of a smallest defining set of $J$, and $\mu_{\lambda}$ be the proportion of blocks in a smallest defining set of $J$. Then $s_{\lambda}=3 \lambda / 2$ and $\mu_{\lambda}=3 / 10$.

Proof: Let $D$ be the unique $2-(6,3,2)$ design. K. Gray [7] has shown that the size of a smallest defining set of $D$ equals three. The results quoted in Gronau [10] imply that $J$ is necessarily decomposable into copies of $D$. By noting that $b=10 \lambda / 2, r=5 \lambda / 2$ and $\lambda_{2}=\lambda$, the result now follows from Lemmas 2.3 and 4.4 .

In general, the lower bound of Lemma 2.3 is not tight, even when all the decomposable designs must be decomposed into copies of a single design. For example, there is a 
single $2-(9,3,1)$ design, with smallest defining set size of $4[7]$. There are $362-(9,3,2)$ designs. These designs, with their properties, are given by Morgan [21] and Mathon and Rosa [18], and the sizes of smallest defining sets are given by Khodkar [15]. From these we see that nine of the $2-(9,3,2)$ designs are decomposable, necessarily into copies of the unique $2-(9,3,1)$ design. However, only six of these nine have smallest defining set sizes of 8 , the other three having sizes of 9 .

We now consider the $2-(7,3, \lambda)$ designs. Grüttmüller notes in [11] that Langdev [17] has shown that there are exactly two indecomposable $2-(7,3, \lambda)$ designs. One of these designs is the $2-(7,3,1)$ design $D$ introduced in Example 1.2 and the other is the 2-(7,3,3) design $I$ introduced in Example 2.2. The number of blocks contained in smallest defining sets of $D$ and $I$ are 3 and 7 respectively; see [7].

Let $\mu$ be the fraction of blocks in a smallest defining set of a $2-(7,3, \lambda)$ design, $J$ say. Now $J$ can be decomposed into isomorphic copies of $I$ and $D$. Lemma 2.3 yields $\mu \geqslant \min (3 / 7,7 / 21)=1 / 3$. By Lemma $4.1, \mu \leqslant 4 / 7$. We now prove a better upper bound, which shows that any $2-(7,3, \lambda)$ design can be defined by less than half its blocks.

THEOREM 4.6. If $D$ is a $2-(7,3, \lambda)$ design, then a smallest defining set has at most $16 \lambda / 5$ blocks.

Proof: Let $V=\{0, \ldots, 6\}$, and suppose that $D=(V, \mathcal{B})$ is a $2-(7,3, \lambda)$ design, with $b=7 \lambda$ and $r=3 \lambda$. Let $\mathcal{B}=X \cup Y$, where $X$ is the set of blocks that do not contain the point 0 . As in the proof of Lemma 4.1, $X$ is a defining set of $D$, in $b-r=4 \lambda$ blocks. We show that $X$ contains a set of at least $4 \lambda / 5$ blocks which can be deleted without destroying its unique completion property.

Consider the $3 \lambda$ blocks in $Y$. These contain a total of $3 \lambda$ pairs from $V \backslash\{0\}$. Since there are $\left(\begin{array}{l}6 \\ 2\end{array}\right)=15$ possible pairs, there must be a pair, say $a b$, that occurs at most $3 \lambda / 15=\lambda / 5$ times in $Y$. Since each pair occurs $\lambda$ times in $D$, there must be at least $4 \lambda / 5$ blocks in $X$ that contain $a b$. Let the set of blocks in $X$ that contain $a b$ be $Z$.

Each element of $V \backslash\{0\}$ has multiplicity $\lambda$ in $Y$, so $X$ is a $1-(6,3,2 \lambda)$ design. Since the blocks of $Z$ all contain the pair $a b, Z$ cannot contain any $(3,1)$ trades. So $X \backslash Z$ is a defining set for $X$. Hence $X \backslash Z$ is a defining set of $D$, and contains at most $4 \lambda-4 \lambda / 5=16 \lambda / 5$ blocks.

Corollary 4.7 . For any $2-(7,3, \lambda)$ design $J, 1 / 3 \leqslant \mu \leqslant 16 / 35$. If $J$ can be decomposed into Fano planes, then $3 / 7 \leqslant \mu \leqslant 16 / 35$.

The $3-(8,4, \lambda)$ designs are self-complementary, and can all be obtained by extending (uniquely) the $2-(7,3, \lambda)$ designs by complementation, as discussed by Khosrovshahi and Vatan [16]. It is easy to see that the extension of an indecomposable 2-(7,3, $\lambda)$ design is an indecomposable $3-(8,4, \lambda)$ design, while any restriction of an indecomposable $3-(8,4, \lambda)$ design is an indecomposable $2-(7,3, \lambda)$ design. So there are precisely two indecomposable $3-(8,4, \lambda)$ designs, one for $\lambda=1$ and one for $\lambda=3$. Let $\mu$ be the proportion of blocks 
in a smallest defining set of a $3-(8,4, \lambda)$ design. Since extension by complementation doubles the number of blocks, the following corollary of Lemma 4.2 and Theorem 4.6 is immediate.

Corollary 4.8. For any $3-(8,4, \lambda)$ design $J, 1 / 6 \leqslant \mu \leqslant 8 / 35$. If $J$ can be decomposed into $3-(8,4,1)$ designs, then $3 / 14 \leqslant \mu \leqslant 8 / 35$.

Note that if $\lambda \leqslant 4$, then Corollaries 4.7 and 4.8 are sufficient to prove the sizes of smallest defining sets for those designs which can be decomposed into Fano planes or into $3-(8,4,1)$ designs.

\section{VARYING $v$}

Let $S_{d}$ be the 2-( $\left(2^{d+1}-1,3,1\right)$ design obtained from the points and lines of $P G(d, 2)$ and let $\mu_{d}$ be the fraction of blocks in a smallest defining set of $S_{d}$. B. Gray [4] observed that $\left\{\mu_{d}\right\}_{d=2}^{\infty}$ is a non-decreasing sequence, and so $\mu_{d} \rightarrow l$ as $d \rightarrow \infty$, for some limiting value $l$. Moran [20] has shown that $\mu_{3}=16 / 35$ and so $l \geqslant 16 / 35$, but the exact value of $l$ is unknown. This is an example of the problem of determining the limit (if it exists) of the size of a smallest defining set as $v$ increases.

Although we make no progress on this particular problem, we do solve for the limiting value of the fraction of blocks in a smallest member defining set for Steiner designs. A simple corollary of this result shows that, asymptotically, the problem of determining the fraction of blocks in a smallest defining set of a Steiner design is equivalent to the problem of finding the fraction of blocks in a smallest class defining set.

LеммA 5.1. Let $\eta_{v}=\left(\log _{2} v !+1\right)\left(\begin{array}{l}k \\ t\end{array}\right) /\left(\begin{array}{l}v \\ t\end{array}\right)$, where $k$ and $t$ are fixed integers with $k>t>1$. Then $\eta_{v} \rightarrow 0$ as $v \rightarrow \infty$.

Proof: Since $k$ and $t$ are fixed, it suffices to show that

$$
\lim _{v \rightarrow \infty} \frac{\log _{2} v !}{\left(\begin{array}{l}
v \\
t
\end{array}\right)}=0 .
$$

Now $\log _{2} v ! \leqslant \log _{2} v^{v}=v \log _{2} v$, and $\left(\begin{array}{l}v \\ t\end{array}\right) \sim v^{t} / t !$. As $t \geqslant 2$, the result follows.

THEOREM 5.2. Define $m_{D}$ to be the size of a smallest member defining set of a Steiner design $D$. Fix $k>t>1$, let

$$
m_{v}=\max \left\{m_{D}: D \text { is a } t-(v, k, 1) \text { design }\right\},
$$

and let $b_{v}$ be the number of blocks in a $t-(v, k, 1)$ design. Then $m_{v} / b_{v} \rightarrow 0$ as $v \rightarrow \infty$.

Proof: Let $D$ be any $t-(v, k, 1)$ design, and let $\mathcal{D}$ be the set of all distinct designs isomorphic to $D$. We present an algorithm to construct a member defining set $M$ of 
size at most $\log _{2} v !+1$ for some $D^{*} \in \mathcal{D}$. Now, using Lemma 5.1 and the fact that $b_{v}=\left(\begin{array}{l}v \\ t\end{array}\right) /\left(\begin{array}{l}k \\ t\end{array}\right)$, we obtain

$$
\frac{m_{v}}{b_{v}} \leqslant \frac{|M|}{b_{v}} \leqslant \frac{\log _{2} v !+1}{b_{v}} \rightarrow 0 \text { as } v \rightarrow \infty .
$$

It remains to construct $M$. Consider the algorithm given in Figure 1. First note that the particular $D^{*}$ for which a member defining set is constructed is irrelevant; in particular, it need not be specified initially. As $|\operatorname{aut}(D)| \geqslant 1$, then $|\mathcal{D}| \leqslant v$ !, and so at most $\log _{2} v !+1$ blocks are sufficient to select $D^{*}$, provided that each choice of block reduces the search-space by at least a half. Now if $|\mathcal{D}| \neq 1$ at $\operatorname{STEP} 1$, then $\mathcal{D}$ must contain at least two distinct designs. Thus the algorithm reaches STEP 5 after a finite number of passes through the loop represented by STEPS $2-4$. Since the chosen $t$-subset $T$ is contained in at least two distinct $B_{i}$, one $B_{i}$ must occur in at most half of the designs in $\mathcal{D}$. Finally, since each $t$-subset is considered at most once the algorithm is guaranteed to terminate, and, when it does, $M$ is a member defining set for $D^{*}$.

REMARK 5.3. Unfortunately, the method of Theorem 5.2 appears unlikely to give a bound on the size of a smallest defining set of a design $D$. For example, Wilson [25] showed that the number of distinct 2- $(v, 3,1)$ designs is at least $\left(e^{-5} v\right)^{v^{2} / 6}$, and $\log _{2}\left(e^{-5} v\right)^{v^{2} / 6}$ dominates $b=v(v-1) / 6$.

Now, if $S$ is a defining set of a design $D$ then $S$ is both a class and a member defining set of $D$. So, for Steiner designs, the problem of finding the asymptotic value for the fraction of blocks in a smallest defining set reduces to the problem of finding the fraction of blocks in a smallest class defining set. Rather than give the general result, we state how the result applies to the designs $S_{d}$ derived from $P G(d, 2)$.

Corollary 5.4. Let $c_{d}$ and $\mu_{d}$ be the fraction of blocks in smallest class defining sets and smallest defining sets respectively of $S_{d}$. Then

$$
\lim _{d \rightarrow \infty} c_{d}=\lim _{d \rightarrow \infty} \mu_{d}=l, \text { for some } l \geqslant \frac{16}{35} .
$$

The method of Theorem 5.2 can be applied to other combinatorial structures. As an example, we consider the problem of finding critical sets of Latin squares.

DEFINITION 5.5: A uniquely completable set in a Latin square $L$ is a partial Latin square which has a unique completion to $L$. A uniquely completable set all of whose proper subsets complete to at least two distinct Latin squares is called a critical set of $L$.

A uniquely completable (respectively, critical) set of a Latin square is conceptually equivalent to a defining (respectively, minimal defining) set of a design. A number of authors have studied the sizes of critical sets in Latin squares; see, for example, the survey paper by Keedwell [14]. It is conjectured that the number of entries in a smallest uniquely completable set of a Latin square of order $n$ is at least $n^{2} / 4$ (respectively, $\left.\left(n^{2}-1\right) / 4\right)$ is $n$ is even (respectively odd). 
FIGURE 1: Algorithm for member defining sets

INPUT: $\quad$ integers $v, k$ and $t$, with $v>k>t>1$;

a $t-(v, k, 1)$ design $D$.

outPuT: a design $D^{*}$ isomorphic to $D$;

a member defining set $M$ for $D^{*}$, with $|M| \leqslant \log _{2} v !+1$.

SETUP: $\quad \mathcal{D} \leftarrow$ set of designs isomorphic to $D$;

$M \leftarrow \emptyset$;

$V_{t} \leftarrow$ set of $t$-subsets of $v$-set.

STEP 1: if $|\mathcal{D}|=1$ then

output the unique $D^{*} \in \mathcal{D}$;

output $M$;

terminate.

STEP 2: pick a $t$-subset $T$ from $V_{t}$.

STEP 3: for each $D_{i} \in \mathcal{D}$ do

$B_{i} \leftarrow$ block in $D_{i}$ containing $T$.

STEP 4: if all the $B_{i}$ are equal then

$V_{t} \leftarrow V_{t} \backslash$ set of $t$-subsets in any $B_{i}$;

goto step 2 .

STEP 5: $B \leftarrow$ a $B_{i}$ that occurs least frequently.

STEP 6: $\quad M \leftarrow M \cup\{B\}$;

$\mathcal{D} \leftarrow$ set of $D_{i} \in \mathcal{D}$ containing $B$;

$V_{t} \leftarrow V_{t} \backslash$ set of $t$-subsets of $B$;

goto step 1 .

An isotopism class of a Latin square $L$ of order $n$ consists of all the Latin squares obtained from $L$ by permuting rows, columns or entries of $L$. So an isotopism class contains at most $(n !)^{3}$ members. Define in the natural way a member uniquely completable set and a class uniquely completable set of a Latin square.

THEOREM 5.6. For a Latin square $L$ of order $n$, let $m_{L}$ be the size of a smallest member uniquely completable set. Let

$$
m_{n}=\max \left\{m_{L}: L \text { is a Latin square of order } n\right\} .
$$

Then $m_{n} / n^{2} \rightarrow 0$ as $n \rightarrow \infty$.

Proof: Using a similar technique to that of Theorem 5.2, one obtains the inequality $m_{n} \leqslant \log _{2}\left((n !)^{3}+1\right)$. It is then simple to verify that the limit is as claimed.

Thus, asymptotically, the problem of determining the size of a smallest uniquely completable set is equivalent to determining the size of a smallest class uniquely completable set. It is hoped that this approach may shed some light on this unsolved problem. 


\section{REFERENCES}

[1] E.J. Billington, 'The intersection problem for combinatorial designs', Congr. Numer. 92 (1993), 33-54.

[2] R.T. Curtis, 'Eight octads suffice', J. Combin. Theory Ser. A 36 (1984), 116-123.

[3] K. Engel, 'The number of indecomposable designs is finite', Ars Combin. 17 (1984), 33-34.

[4] B.D. Gray, 'Smallest defining sets of designs associated with $P G(d, 2)$ ', Australas. J. Combin. 16 (1997), 87-98.

[5] B.D. Gray, N. Hamilton and C.M. O'Keefe, 'On the size of a smallest defining set of $P G(2, q)$ ', Bull. Inst. Combin. Appl. 21 (1997), 91-94.

[6] K. Gray, 'On the minimum number of blocks defining a design', Bull. Austral. Math. Soc. 41 (1990), 97-112.

[7] K. Gray, 'Further results on smallest defining sets of well known designs', Australas. J. Combin. 1 (1990), 91-100.

[8] K. Gray, 'Defining sets of single-transposition-free designs', Utilitas Math. 38 (1990), 97-103.

[9] C.S. Greenhill and A.P. Street, 'Smallest defining sets of some small $t$-designs and relations to the Petersen graph', Utilitas Math. 48 (1995), 5-31.

[10] H-D.O.F. Gronau, 'A survey of results on the number of $t-(v, k, \lambda)$ designs.', Ann. Discrete Math. 26 (1985), 209-220.

[11] M. Grüttmüller, 'On the number of indecomposable block designs', Australas. J. Combin. 14 (1996), 181-186.

[12] A. Hedayat, 'The theory of trade-off for $t$-designs', in Coding theory and design theory, part II: Design theory, (D. Raychaudhuri, Editor), IMA Volumes in Mathematics and its Applications 21 (Springer-Verlag, Berlin, Heidelberg, New York, 1990), pp. 101-126.

[13] H.L. Hwang, 'On the structure of $(v, k, t)$ trades', J. Statist. Plann. Inference 13 (1986), 179-191.

[14] A.D. Keedwell, 'Critical sets for Latin squares, graphs and block designs: a survey', Congr. Numer. 113 (1996), 231-245.

[15] A. Khodkar, 'Smallest defining sets for the 36 non-isomorphic twofold triple systems of order nine', J. Combin. Math. Combin. Comput. 17 (1994), 209-215.

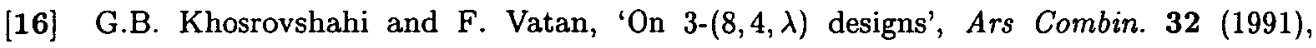
115-120.

[17] I. Langdev, 'Nondecomposable block designs with $v=7$ and $\lambda=3$ ', in Mathematics and mathematical education (Bulgarian) (Albena, April 6-10, 1989), (G. Gerov, Editor) (Bulgarian Academy of Sciences, Sofia, 1989), pp. 386-389.

[18] R. Mathon and A. Rosa, 'A census of Mendelsohn triple systems of order nine', Ars Combin. 4 (1977), 309-315.

[19] R. Mathon and A. Rosa, '2- $(v, k, \lambda)$ designs of small order', in CRC Handbook of Combinatorial Designs, (C.J. Colbourn and J.H. Dinitz, Editors) (CRC Publishing Co., Boca Raton, Fl., 1996), pp. 3-41.

[20] A.T. Moran, Block designs and their defining sets, (Ph.D. Thesis) (The University of Queensland, Australia, 1997).

[21] E.J. Morgan, 'Some small quasi-multiple designs.', Ars Combinatoria 3 (1977), 233-250. 
[22] D. Sarvate and J. Seberry, 'A note on small defining sets for some $S B I B D(4 t-1,2 t-1, t-1)$ ', Bull. Inst. Combin. Appl. 10 (1994), 26-32.

[23] A.P. Street, 'Defining sets for block designs: an update', in Combinatorics advances, (C.J. Colbourn and E.S. Mahmoodian, Editors) (Kluwer Academic Press, Norwell, MA, 1995), pp. 307-320.

[24] A.P. Street, 'Trades and defining sets', in CRC Handbook of Combinatorial Designs, (C.J. Colbourn and J.H. Dinitz, Editors) (CRC Publishing Co., Boca Raton, Fl, 1996), pp. 474-478.

[25] R.M. Wilson, 'Nonisomorphic Steiner triple systems', Math. Z. 135 (1974), 303-313.

Department of Mathematics

The University of Queensland

Queensland 4072 Australia

e-mall: bdg@matns.uq.eau.au
Centre for Discrete Mathematics and Computing The University of Queensland Queensland 4072 Australia e-maii: cramw̄csee.uq.ecu.du 